\title{
Anesthetic Management of a Child From the Qinghai-tibet Plateau With a Double Outlet Right Ventricle and Severe Polycythemia: A Case Report
}

\section{Lingcan Tan}

Sichuan University West China Medical Center

\section{Weiyi Zhang}

Sichuan University West China Hospital

\section{Yiding Zuo}

Sichuan University West China Hospital

\section{Hongyang Chen}

Sichuan University West China Hospital

Chunling Jiang ( $\nabla_{\text {jiangchunling@scu.edu.cn ) }}$

Sichuan University West China Hospital

\section{Case report}

Keywords: cyanotic congenital heart diseases, double outlet right ventricle, modified Fontan surgery, erythrocytosis, coagulopathy, hemodilution

Posted Date: July 28th, 2020

DOI: https://doi.org/10.21203/rs.3.rs-47461/v1

License: (c) (1) This work is licensed under a Creative Commons Attribution 4.0 International License. Read Full License 


\section{Abstract \\ Background}

Acquired secondary polycythemia is common in patients with a double outlet right ventricle, and results from tissue hypoxia. Secondary erythrocytosis can cause coagulation deficiencies, and increases the risks of thrombosis and infarction. Perioperative management in double outlet right ventricle patients with severe erythrocytosis is intractable, and only a few cases have been reported.

\section{Case presentation:}

Herein, we report the anesthetic management of a 10-year-old female patient with a double outlet right ventricle. She lived in the low-oxygen Qinghai-Tibet Plateau, and presented with severe polycythemia (hemoglobin, $24.8 \mathrm{~g} / \mathrm{dL}$; hematocrit, 75\%). She underwent a modified Fontan surgery, which was satisfactory and without any perioperative complications. Our anesthetic management highlights the importance of perioperative hemodilution to decrease the risk of thromboembolism, and correction of coagulopathy to prevent hemorrhage.

\section{Conclusions}

Anesthetic management is challenging in rare cyanotic congenital heart disease patients with severe polycythemia. For prevention of thrombosis and hemorrhage, it is important to adopt perioperative hemodilution and correction of coagulopathy.

\section{Introduction}

Erythrocytosis is characterized by increased levels of red blood cells under the control of erythropoietin, and is frequently observed in patients with congenital heart disease. However, severe decompensated erythrocytosis dramatically increases the risks of thrombosis, infarction, and coagulation disorders. Cases of cyanotic congenital heart disease (CCHD) with severe polycythemia and a hemoglobin $(\mathrm{Hb})$ level $\geq 24 \mathrm{~g} / \mathrm{dL}$ are rare. Herein, we present a case of 10-year-old girl from the Qinghai-Tibet Plateau with a double outlet right ventricle (DORV) and an elevated $\mathrm{Hb}$ level of $24.8 \mathrm{~g} / \mathrm{L}$, who underwent a modified Fontan operation. Written authorization for the case report was obtained from the patient's family.

\section{Case Presentation}

A 10-year-old girl from the Liangshan prefecture in Sichuan Province, Qinghai-Tibet Plateau, China, presented to hospital with cardiac murmurs for 6 years. She was diagnosed with DORV and advised to undergo surgical treatment 6 years ago. However, she was unable to have the surgery because of economic reasons. Her situation progressive worsened, and she was eventually admitted to our center. 
Her physical examination revealed delayed growth (height, $119 \mathrm{~cm}$; weight, $19 \mathrm{~kg}$ ), a blood pressure of $94 / 65 \mathrm{mmHg}$, a pulse rate of 98 beats per min, and a respiratory rate of 24 breaths/min. Her baseline oxygen saturation is maintained at about $80 \%$. Blood tests showed a $\mathrm{Hb}$ of $24.8 \mathrm{~g} / \mathrm{dL}$, hematocrit (Hct) of $75 \%$, mean corpuscular volume of $93.3 \mathrm{fL}$, mean corpuscular $\mathrm{Hb}$ of $31 \mathrm{pg}$, mean corpuscular $\mathrm{Hb}$ concentration of $33.2 \mathrm{~g} / \mathrm{dL}$, and a platelet count of $108 \times 10^{9} \mathrm{cells} / \mathrm{L}$. Her blood coagulation test was also abnormal, with a prothrombin time of $16.7 \mathrm{~s}$, active partial thrombin time of $61.8 \mathrm{~s}$, and an international normalized ratio of 1.51 . Other blood tests showed no significant abnormalities. Transthoracic echocardiography (TTE) revealed a DORV, a ventricular septal defect (VSD) with a bidirectional shunt at the ventricular level, an atrial septal defect (ASD) with a right-to-left shunt at the atrial level, severe pulmonary valve stenosis, right ventricular hypertrophy, and transposition of the great arteries, but with normal left ventricular functions (ejection fraction, 66\%) (Fig. 1). Chest radiography demonstrated abnormal cardiac morphology (Fig. 2), while chest computed tomography showed a DORV with a VSD, an ASD, subvalvular pulmonary artery stenosis and transposition of the great arteries. (Fig. 3). Right cardiac catheterization was performed after admission. Angiography showed normal distal pulmonary artery development, and multiple aorta pulmonary collateral arteries, two of which were successfully occluded during the procedure. The mean pulmonary artery pressure was measured as $13 \mathrm{mmHg}$. Considering normal distal pulmonary artery development, normal left ventricular function and acceptable pulmonary vascular resistance (PVR), a modified Fontan operation was then planned.

\section{Anesthesia And Surgery}

General anesthesia was selected because of the patient's severe and complicated condition. Routine monitoring was performed. After anesthesia, induction was performed with sufentanil $(12.5 \mu \mathrm{g})$, midazolam $(1.5 \mathrm{mg})$, and cisatracurim $(6 \mathrm{mg})$, and the patient was smoothly intubated without a marked decrease in oxygen saturation. The radial artery and internal jugular vein were then catheterized to monitor blood pressure and central venous pressure (CVP), respectively. Continuous transesophageal echocardiography (TEE) examination was performed to assess cardiac function and volume status throughout the procedure (Fig. 4). Anesthesia was maintained with sevoflurane (1\%-3\%), propofol (2-6 $\mathrm{mg} / \mathrm{kg} / \mathrm{h})$, and remifentanil (0.1-0.2 $\mu \mathrm{g} / \mathrm{kg} / \mathrm{min})$. Intraoperative mechanical ventilation strategies included a tidal volume of $6-8 \mathrm{mg} / \mathrm{kg}$, and maintaining an end-tidal $\mathrm{CO}_{2}$ between $25-30 \mathrm{mmHg}$.

After induction, preoperative hemodilution was performed by infusion of crystalloid solution $(350 \mathrm{~mL})$ over $1 \mathrm{~h}$ to decrease blood viscosity and prevent thrombotic complications. Tranexamic acid was administered before incision to inhibit the fibrinolytic process and prevent bleeding. Crystalloid solution and human albumin was also added to priming solution used for cardiopulmonary bypass (CPB) to further dilute circulating red blood cells. Hct was maintained at $45-55 \%$ in the CPB. A fenestration modified Fontan operation was performed. After performing a total cavapulmonary connection, the surgeon implemented a right atrium fenestration about 3 millimeter. The surgical procedure was uneventful. The left atrium was then catheterized to continuously assess left atrial pressure prior to discontinuing CPB. After discontinuing CPB, sufficient blood volume (based on dynamic TEE monitoring 
and blood gas analysis) and a higher CVP were maintained to provide adequate preload for the right ventricle, to facilitate blood flow towards the pulmonary circulation. The patient received $670 \mathrm{~mL}$ of crystalloid solution, $200 \mathrm{~mL}$ of plasma, 1 unit of platelets, and a $300-\mathrm{mL}$ transfusion of autologous recovered blood.

The patient was then shifted to the intensive care unit (ICU). Fortunately, postoperative bleeding was mild and she continuously administered plasma and platelets to correct coagulation disorder. Changes in $\mathrm{Hb}$ levels and coagulative function are shown in Table 1. In ICU, the patient was placed in a head-high position to facilitate vena cava reflux. Tracheal tube was extubated on postoperative day 1 , and she was adopted thoracentesis and drainage on postoperative day 2. Considering that she was in a stable condition and no complications occurred, the patient was transferred to general ward on postoperative day 4 and was discharged on postoperative day 7 .

Table 1

The variation of $\mathrm{Hb}$ and coagulation function

\begin{tabular}{|lllll|}
\hline Variables & Preoperative & Postoperative day $\mathbf{1}$ & $\begin{array}{l}\text { Postoperative } \\
\text { day } \mathbf{4}\end{array}$ & $\begin{array}{l}\text { Postoperative } \\
\text { day } \mathbf{8}\end{array}$ \\
\hline $\mathrm{Hgb}(\mathrm{g} / \mathrm{dL})$ & 24.8 & 16.7 & 15.7 & 15.3 \\
\hline $\mathrm{PT}(\mathrm{s})$ & 16.7 & 18.6 & 29.7 & 15.5 \\
\hline INR & 1.51 & 1.70 & 2.77 & 1.4 \\
\hline APTT (s) & 61.8 & 56.9 & 51.6 & 43.8 \\
\hline
\end{tabular}

\section{Discussion}

Perioperative management of polycythemic CCHD patients undergoing complex cardiac surgery requires adequate planning and careful implementation. The present study demonstrates the successful anesthetic management of a rare DORV patient with severe polycythemia who underwent a modified Fontan surgery. The patient showed a favorable postoperative course without any complications. In this case, hemodilution and management of coagulation functions were key goals.

Because of chronic slow progressive hypoxemia and central cyanosis, patients with DORV may experience serious erythrocytosis and coagulation disorders. Additionally, our patient had lived in the Qinghai-Tibet Plateau in China for 10 years. The low-oxygen environment of the plateau may have also contributed to the severe erythrocytosis ( $\mathrm{Hb}$ levels up to $24.8 \mathrm{~g} / \mathrm{dL}$ ). Under such conditions, thrombosis and infarction can be clinically-devastating complications. However, phlebotomy is not recommended for the prevention of cerebrovascular events, as it can cause iron deficiency, reduce exercise tolerance, and impair oxygen transport capacity ${ }^{[1]}$. In the present case, we performed hemodilution rather than phlebotomy to ameliorate hyperviscosity. Crystalloid solution was administrated to maintain blood volume and decrease blood viscosity from beginning of anesthesia. During extracorporeal circulation, 
hemodilution was also adopted throughout. Intraoperative volume management was constantly regulated according to dynamic TEE monitoring and blood gas analysis to maintain the balance between adequate blood viscosity and appropriate volume load. Sahoo et al showed that hemodilution in CCHD patients has beneficial effects including improved shunt patency and less postoperative blood loss, and it is safe to reduce Hct to $45 \%$ [2].

Coagulation dysfunction is also common in cyanotic patients, and involves a multi-systemic mechanism including thrombocytopenia, shortened platelet survival, and deficiencies in coagulation factors ${ }^{[3-9]}$. Therefore, an individualized intraoperative anticoagulation and antifibrinolytics strategy should be adopted for children with cyanosis. As an alternative treatment, measuring preoperative antithrombin activity and supplementing its activity prior to CPB can preserve the efficacy of heparin during CPB. In addition, epsilon aminocaproic acid and tranexamic acid are two widely available lysine analogs used to inhibit the fibrinolytic process to reduce clinical bleeding ${ }^{[10]}$. In the present case, postoperative management of coagulation functions was refractory and tenacious. Her coagulopathy suggested an increased risk of postoperative hemorrhage, for another, anticoagulation should be implemented as soon as possible after surgery to prevent thromboembolic complications. The coagulation disorders were corrected until well after surgery following repeated transfusions. Thromboelastography (TEG) can also be used to monitor perioperative coagulation function for TEG can substantiate changes in coagulation status produced by hemodilution. TEG measurements have become an accepted measure to assess changes in coagulation after volume replacement ${ }^{[11-12]}$.

Maintenance of perioperative hemodynamics is also vital to the success of surgery. Adequate preoxygenation, proper sedation, and prevention of hypoxic crisis before induction should be implemented. Perioperative fluid management is critical for adequate volume control, and is required to maintain sufficient CVP to facilitate blood flow towards the pulmonary circulation while maintaining sufficient colloid osmotic pressure. After $\mathrm{CPB}$, maintaining low pulmonary vascular resistance while maintaining a high right atrial pressure and a low left atrial pressure are key for hemodynamic stability

[13-14]. Hypercapnia must be prevented, and any drugs that may increase PVR should be avoided. Adequate hyperventilation and use of positive vasoactive agents can enhance myocardial systole, decrease PVR, and maintain cardiac output. Postoperatively, we ensure that patients are smoothly adapted to their unique Fontan circulation hemodynamics. Since positive pressure mechanical ventilation increases intra-alveolar pressure, which can increase PVR and decrease cardiac output, positive endexpiratory pressure can be avoided and the lowest possible inspiratory pressure should be maintained.

\section{Conclusions}

We presented the anesthetic management of a high-risk CCHD patient with severe polycythemia who underwent a modified Fontan surgery. Our anesthetic management highlights the importance of hemodilution for ameliorating hyperviscosity to decrease the risk of thromboembolism. Furthermore, it is equally important to correct coagulopathy to prevent hemorrhage. Maintenance of stable intraoperative 
hemodynamics to help the patient adapt smoothly to their unique Fontan circulation is also vital to the success of the surgery.

\section{Abbreviations}

CCHD

cyanotic congenital heart disease; $\mathrm{Hb}$; hemoglobin; DORV:double outlet right ventricle; Hct:hematocrit; VSD:ventricular septal defect; ASD:atrial septal defect; PVR:pulmonary vascular resistance; TTE:transthoracic echocardiography; CVP:central venous pressure; TEE:transesophageal echocardiography; CPB:cardiopulmonary bypass; ICU:intensive care unit; TEG:Thromboelastography; RA, right atrium; RV, right ventricle; TV, tricuspid valve; Ao, aorta; PA, pulmonary artery. $\mathrm{PT}$, prothrombin time; APTT, active partial thrombin time; INR, international normalized ratio.

\section{Declarations}

\section{Acknowledgments}

We thank LiwenBianji, Edanz Editing China (www.liwenbianji.cn/ac), for editing the English text of a draft of this manuscript.

\section{Funding}

This researchwas funded by Research Grant from the 1.3.5. project for disciplines of excellence(2018HXFH046),West China Hospital, Sichuan University andthe National Natural Science Foundation ofChina (81971806).

\section{Authors' contributions}

LCT and CLJ were the principal author and principal investigator. WYZ, HYC and YDZ helpedwriting the case report as co-authors. All authors read and approved the final manuscript.

\section{Conflicts of Interest}

The authors have no conflicts of interest to declare.

\section{Availability of data and materials}

Not applicable.

\section{Ethics approval and consent to participate}

This case report was approved by the institution review board.

\section{Consent for publication}


Written informed consent for publication of the clinical details was obtained from the patient.

\section{References}

1. Diller GP, Dimopoulos K, Broberg CS, et al. Presentation, survival prospects, and predictors of death in Eisenmenger syndrome: a combined retrospective and case-control study. Eur Heart J. 2006;27(14):1737-42.

2. Sahoo TK, Chauhan S, Sahu M, et al. Effects of Hemodilution on Outcome After Modified BlalockTaussig Shunt Operation in Children With Cyanotic Congenital Heart Disease[J]. Journal of Cardiothoracic Vascular Anesthesia. 2007;21(2):179-83.

3. Perloff JK, Rosove MH, Sietsema KE, et al. Cyanotic congenital heart disease: a multisystem disorder. In: Congenital Heart Disease in Adults. Philadelphia: W.B Saunders Company; 1998. pp. 199-226.

4. Maurer HM, McCue CM, Robertson JC, et al. Correction of platelet dysfunction and bleeding in cyanotic congenital heart disease by simple red cell volume reduction. Am J Cardiol. 1975;35:831-5.

5. Niwa K, Perloff JK, Kaplan S, et al. Eisenmenger syndrome in adults. Ventricular septal defect, truncus arteriosus, and univentricular heart. J Am Coll Cardiol. 1999;34:223-32.

6. Territo MC, Perloff JK, Rosove MH, et al. Acquired von Willebrand factor abnor- malities in adult with congenital heart disease: Dependence Upon Cardiopulmonary Pathophysiological Subtype. Clin Appl Thromb Hemost. 1998;4:257-61.

7. Peters AM, Rozkocev A, Bell RN, et al. Platelet kinetics in congenital heart disease. Cardiovasc Res. 1982;16:391-7.

8. Heriksson P, Varendh G, Lundstrom NR. Haemostatic defects in cyanotic congenital heart disease. $\mathrm{Br}$ Heart J. 1979;41:23-7.

9. Levin E, Wu J, Devine DV, et al. Hemostatic parameters and platelet activation marker expression in cyanotic and acyanotic padiatric patients undergoing cardiac surgery in the presence of tranexamic acid. Thromb Haemost. 2000;83:54-9.

10. Zabala LM, Guzzetta NA. Cyanotic congenital heart disease (CCHD): focus on hypoxemia, secondary erythrocytosis, and coagulation alterations. Paediatr Anaesth. 2015;25(10):981-9. DOI:10.1111/pan.12705.

11. Karoutsos S, Nathan N, Lahrimi A, et al. Thrombelastogram reveals hypercoagulability after administration of gelatin solution.[J]. British Journal of Anaesthesia(2):175-177.

12. Phillips Ryan C,Shahi Niti,Leopold David et al. Thromboelastography-guided management of coagulopathy in neonates with congenital diaphragmatic hernia supported by extracorporeal membrane oxygenation.[J].Pediatr. Surg. Int., 2020, undefined: undefined.

13. Ohuchi H. Where Is the"Optimal”Fontan Hemodynamics? Korean Circulation Journal, 2017, 47(6).

14. Levine TB, Levine AB. Regional blood flow supply and demand in heart failure. Am Heart J. 1990;120:1547-51. 


\section{Figures}
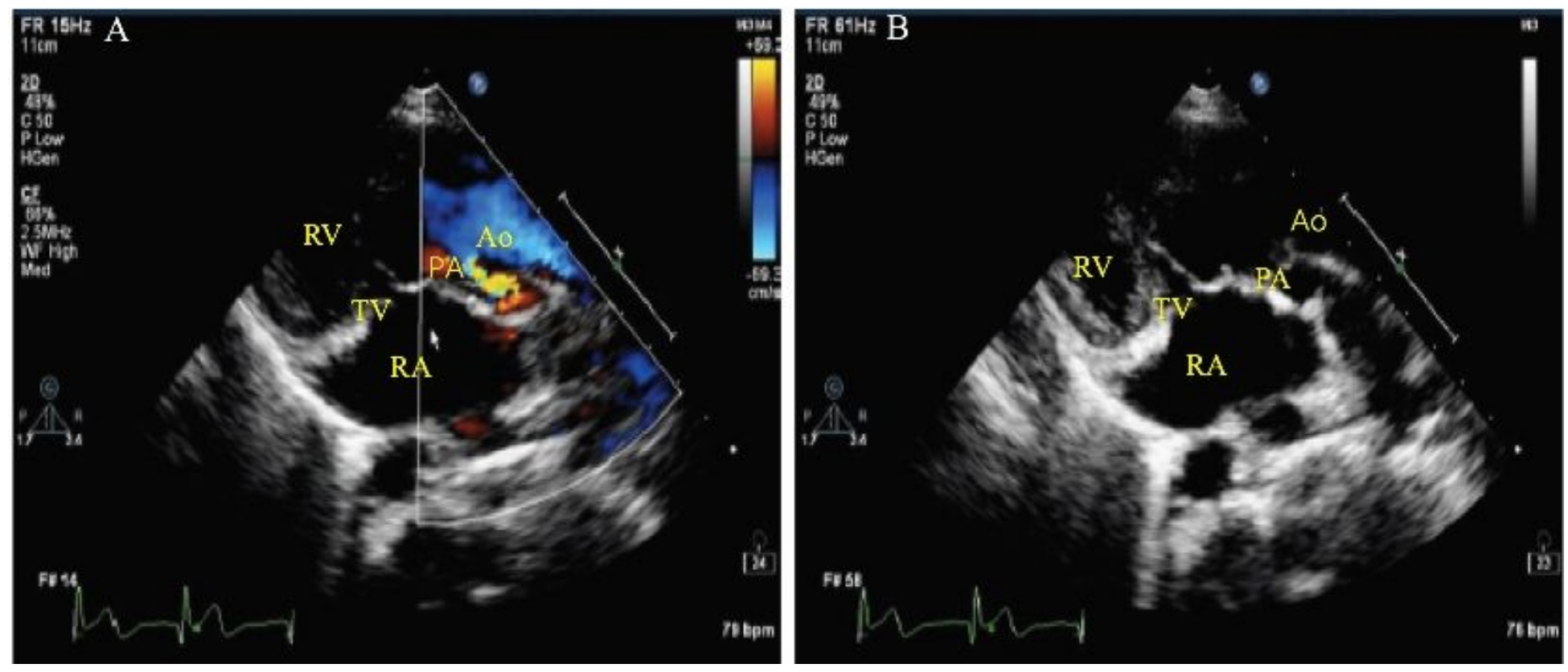

\section{Figure 1}

Preoperative TTE showed DORV and pulmonary artery stenosis.RA, right atrium; RV, right ventricle; TV, tricuspid valve;Ao, aorta; PA, pulmonary artery; DORV, double outlet right ventricle;TTE, transthoracic echocardiography.

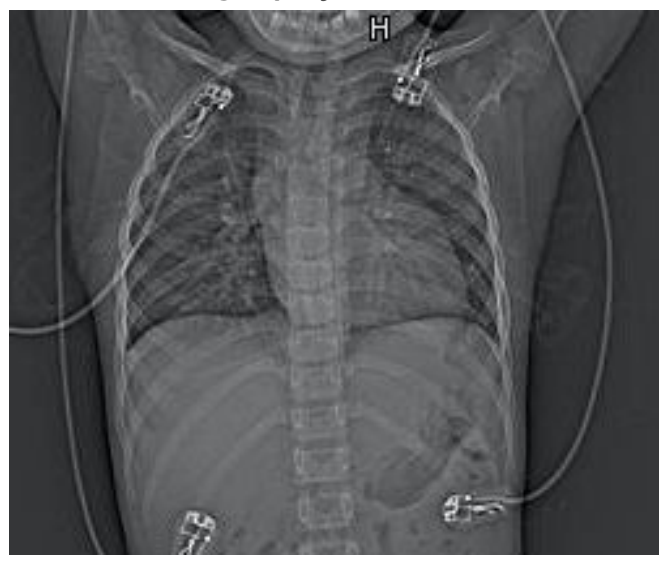

\section{Figure 2}

Chest radiography demonstrated abnormal cardiac morphology. 

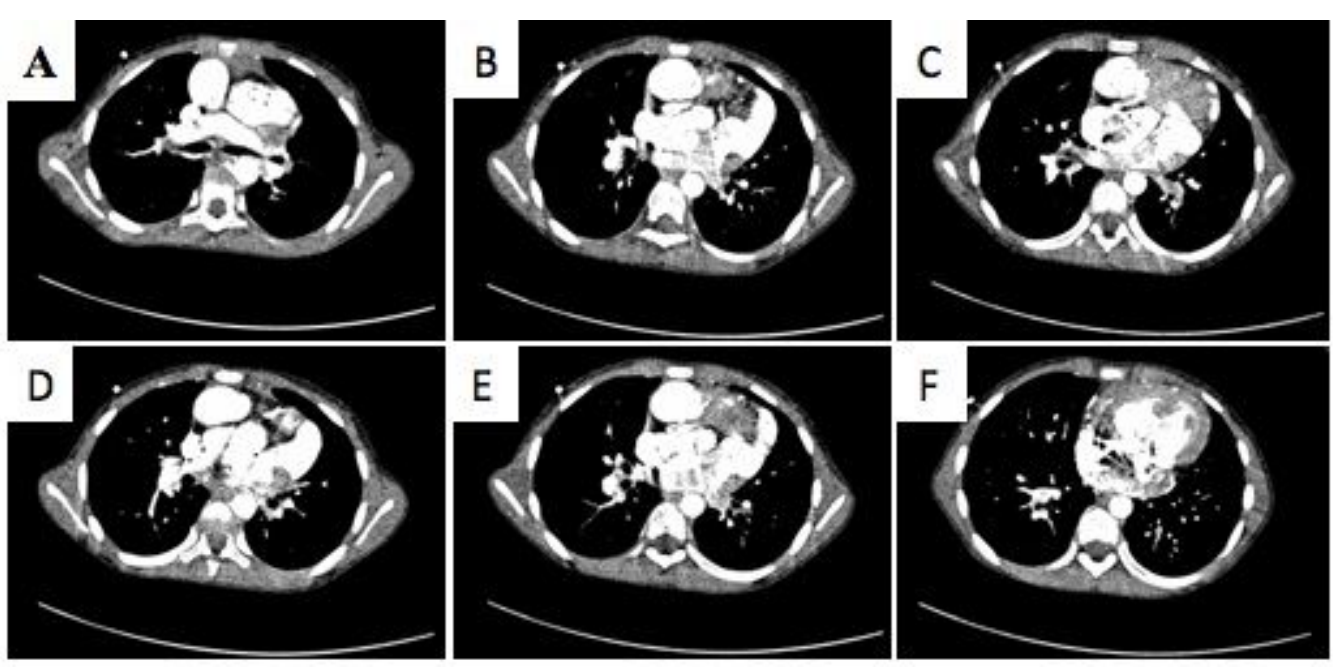

Figure 3

Chest computed tomography. $(\mathrm{A}] \mathrm{C})$ Both the aorta and pulmonary artery were connected to the right ventricle, and the aorta was anterior to the pulmonary artery. (DIF) Significant right ventricular enlargement, ASD (width, $1.4 \mathrm{~cm}$ ), and VSD (width, $1.7 \mathrm{~cm}$ ).

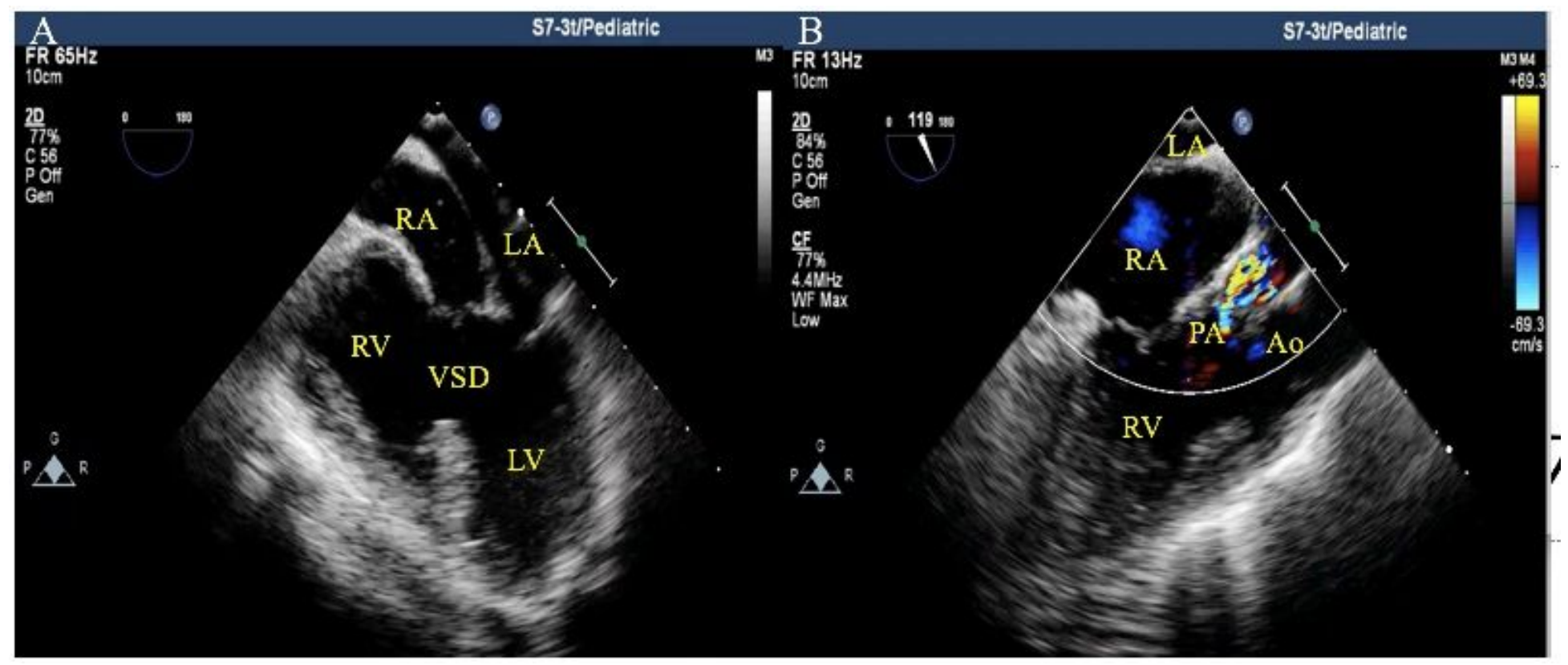

\section{Figure 4}

Intraoperative TEE. (A) VSD was evident. (B) Both the aorta and the pulmonary artery evolved from the right ventricle, with significant pulmonary artery stenosis.LA, left atrium; LV, left ventricle; RA, right atrium; $\mathrm{RV}$, right ventricle; Ao, aorta; PA, pulmonary artery; VSD, ventricular septal defect;TEE, transoesophageal echocardiogram.

\section{Supplementary Files}

This is a list of supplementary files associated with this preprint. Click to download. 
- CAREchecklistEnglish20132.pdf

- TEE2.avi

- TEE1.avi 